\title{
RpoS induces expression of the Vibrio anguillarum quorum-sensing regulator VanT
}

\author{
Barbara Weber, Antony Croxatto, $†$ Chang Chen $\ddagger$ and Debra L. Milton \\ Department of Molecular Biology, Umeå University, S-901 87 Umeå, Sweden
}

\begin{abstract}
Correspondence
Debra L. Milton

Debra.Milton@molbiol.umu.se
\end{abstract}

Received 17 October 2007

Revised 14 December 2007

Accepted 20 December 2007
In vibrios, regulation of the Vibrio harveyi-like LuxR transcriptional activators occurs posttranscriptionally via small regulatory RNAs (sRNAs) that destabilize the luxR mRNA at a low cell population, eliminating expression of LuxR. Expression of the sRNAs is modulated by the vibrio quorum-sensing phosphorelay systems. However, vanT mRNA, which encodes a LuxR homologue in Vibrio anguillarum, is abundant at low and high cell density, indicating that VanT expression may be regulated via additional mechanisms. In this study, Western analyses showed that VanT was expressed throughout growth with a peak of expression during late exponential growth. VanO induced partial destabilization of vanT mRNA via activation of at least one Orr sRNA. Interestingly, the sigma factor RpoS significantly stabilized vanT mRNA and induced VanT expression during late exponential growth. This induction was in part due to RpoS repressing expression of Hfq, an RNA chaperone. RpoS is not part of the quorum-sensing regulatory cascade since RpoS did not regulate expression or activity of VanO, and RpoS was not regulated by VanO or VanT. VanT and RpoS were needed for survival following UV irradiation and for pigment and metalloprotease production, suggesting that RpoS works with the quorum-sensing systems to modulate expression of VanT, which regulates survival and stress responses.

\section{INTRODUCTION}

Bacteria coordinate activities as a population through a type of cell-cell communication called quorum sensing (Waters \& Bassler, 2005). Small diffusible signal molecules, such as $\mathrm{N}$-acylhomoserine lactones, are secreted by the bacteria and accumulate as the population grows. When a signal molecule reaches a threshold concentration at a critical cell density, quorum-sensing systems are activated, inducing or repressing phenotypic expression. Consequently, bacteria coordinate activities as a population, which likely provides a selective advantage in the natural environment. First characterized as a means for regulating light production in the marine bacteria Vibrio fischeri and Vibrio harveyi, quorum sensing is now recognized as a widespread mechanism for global gene regulation in many bacteria.

Vibrio anguillarum is widely distributed in the aquatic environment and is part of the normal microflora of marine fish (Austin \& Austin, 1999; Urakawa \& Rivera,

tPresent address: Center for Research on Intracellular Bacteria (CRIB), Institute for Microbiology, University of Lausanne, Switzerland.

$\ddagger$ Present address: Guangdong Province, South China Sea Institute of Oceanology, Xinggang Xi Lu, 164, 510301 Guangzhou, China.

Abbreviations: qRT-PCR, quantitative reverse transcriptase polymerase chain reaction; sRNA, small regulatory RNA.

The GenBank/EMBL/DDBJ accession numbers for the sequences of rpoS and $h f q$ are EU330190 and EU330191, respectively.
2006). When the health or immune system of the fish is compromised, $V$. anguillarum causes a haemorrhagic septicaemia (vibriosis) (Actis et al., 1999; Austin \& Austin, 1999). Production of quorum-sensing signal molecules such as $\mathrm{N}$-acylhomoserine lactones is a common feature of both pathogenic and environmental isolates of $V$. anguillarum. Thus, quorum sensing may affect the ecology and physiology of this bacterium as well as its pathogenicity (Buch et al., 2003). Moreover, the V. harveyi-like LuxR transcriptional activator in $V$. anguillarum, VanT, positively regulates extracellular protease activity, pigment production and biofilm formation in response to quorum-sensing signals (Croxatto et al., 2002). Each of these activities may play a role in the survival of $V$. anguillarum in seawater or in the fish host.

All vibrios analysed so far contain quorum-sensing systems involving phosphorelay systems that are believed to regulate gene expression in response to cell population similarly to the quorum-sensing systems of $V$. harveyi (Waters \& Bassler, 2005; Neiditch et al., 2005; Timmen et al., 2006; Tu \& Bassler, 2007). Components of two phosphorelay quorum-sensing systems are known in $V$. anguillarum and a third is predicted. A model of these signalling systems is given in Fig. 1(a). VanM, an $\mathrm{N}$ acylhomoserine lactone synthase, synthesizes the signal molecules $N$-hexanoyl-L-homoserine lactone (C6-HSL) and $\mathrm{N}$-(3-hydroxyhexanoyl)-L-homoserine lactone (3hydroxy-C6-HSL), which are sensed by VanN (Croxatto 
(a)

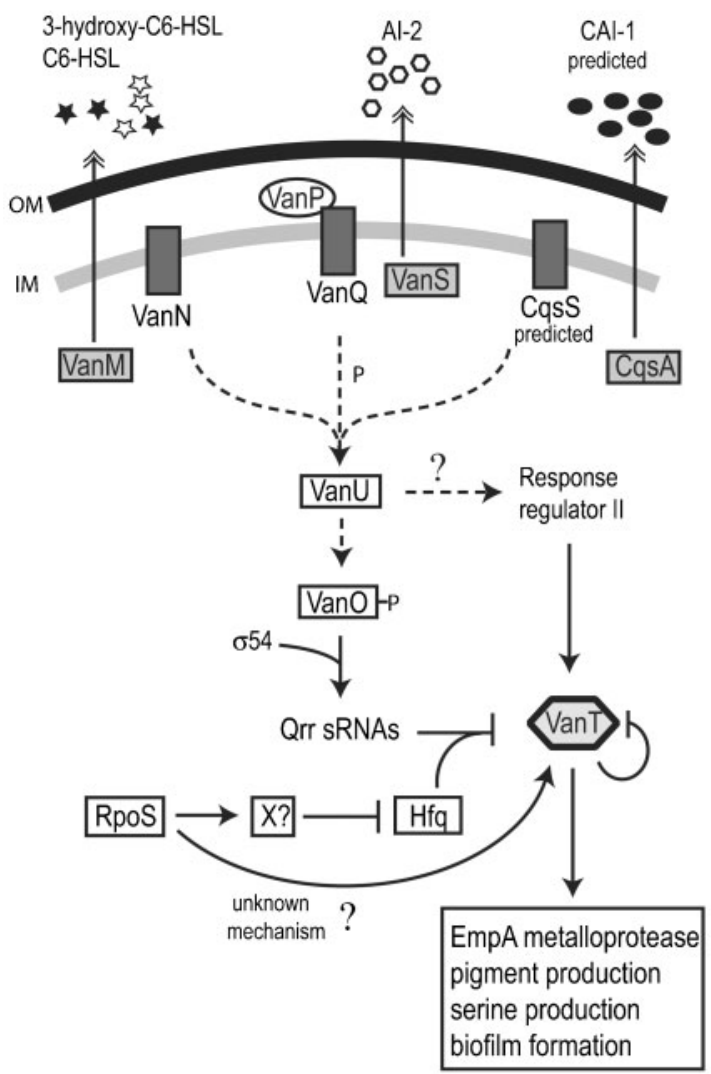

(b)

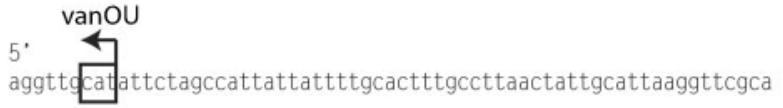

ttttgcaaaataaaataacaaaaaggcactaattcgttagttaatagcccttttgcataaaa ttaattggcacgatatgtgctctattcaatatgacccttaggggtcacctagccaactgacgt

terminator

tgttagtgaataaagtgtcacaaataacagccaatagaccttttctattggctatttt

et al., 2004). VanS likely synthesizes an AI-2 signal molecule, a furanosyl borate diester, which binds the periplasmic protein VanP. The VanP-AI-2 complex then binds to VanQ (Croxatto et al., 2004; Denkin \& Nelson, 2004). The CqsA/S signal system has not been characterized but is predicted to be present (Henke \& Bassler, 2004). The CAI-1 signal molecule, an (S)-3-hydroxytridecan-4-one (Higgins et al., 2007), is synthesized by CqsA and is believed to bind the receptor CqsS.

The three receptor proteins, VanN, VanQ and CqsS, are hybrid sensor kinases that channel information via a phosphorylation cascade to a single regulatory pathway. At low cell densities, in the absence of signal molecules, VanN,
Fig. 1. (a) Model for quorum sensing in $V$. anguillarum. See the text for an explanation. Double arrowheads indicate synthesis and transport of signal molecules to the external environment. Arrows with dashed lines indicate the relay of phosphoryl groups from one protein to another. Arrows with solid lines indicate activation of gene expression and a solid line with a crossing line indicates repression of gene expression. IM, inner membrane; OM, outer membrane. (b) Genomic organization of the qrr1 (quorumregulatory RNA) gene. The shaded sequence indicates the predicted qrr1 gene based on the prediction of Lenz et al. (2004). The rightward-pointing arrow indicates the predicted start site of $q r r 1$ and the terminator sequence is labelled. The predicted $\sigma^{54}-24 /-12$ promoter sequences are labelled and agree with the consensus sequence YTGGCACG- $\mathrm{N}_{4}$-TTGCW (Barrios et al., 1999). The start codon for the divergently transcribed vanOU genes is indicated by a block and a leftward arrow.

VanQ and CqsS act as kinases relaying phosphoryl groups to the phosphotransferase VanU, which phosphorylates the $\sigma^{54}$-dependent activator VanO. When phosphorylated, $\mathrm{VanO}$ activates the expression of several small regulatory RNAs (sRNAs) that, together with the RNA chaperone Hfq, destabilize mRNA encoding VanT, the master regulator. At high cell density, the signal molecules accumulate and bind their cognate sensor kinases. Signal binding represses the kinase activity of VanN, VanQ and CqsS, allowing the phosphatase activity to predominate, which leads to dephosphorylation of VanO. Consequently, VanO is inactivated, sRNAs are not transcribed, and VanT expression is induced, activating the quorum-sensing regulon.

In this study, the protein profile of VanT expression was analysed during growth of wild-type $V$. anguillarum. VanT expression could be detected at a population of $3 \times 10^{6}$ cells $\mathrm{ml}^{-1}$ and the expression peaked as the cells entered late exponential growth. Interestingly, the sigma factor RpoS was shown to play a major role in indirectly inducing VanT expression post-transcriptionally. RpoS stabilizes vanT mRNA by a mechanism involving Hfq. RpoS was not part of the quorum-sensing regulatory cascade since it did not regulate expression or activity of VanO and since RpoS was not regulated by the quorum-sensing systems. Finally, VanT and RpoS were needed for survival following UV irradiation and for pigment and metalloprotease production. In summary, RpoS and the quorum-sensing systems work together to modulate expression of VanT, which regulates physiological responses required for survival and stress response.

\section{METHODS}

Strains, plasmids and media. Bacterial strains and plasmids are described in Table 1. Plasmid transfers from Escherichia coli to V. anguillarum were done as previously described (Milton et al., 1996). E. coli was routinely grown in Luria broth (per litre: Bacto Tryptone, $10 \mathrm{~g}$; Bacto yeast extract, $5 \mathrm{~g}$; and sodium chloride, $5 \mathrm{~g}$ ) with the following antibiotic concentrations: $100 \mu \mathrm{g} \mathrm{ml}^{-1}$ for ampicillin and 
Table 1. Bacterial strains and plasmids

\begin{tabular}{|c|c|c|}
\hline Strain or plasmid & Genotype or relevant markers & Reference or source \\
\hline \multicolumn{3}{|l|}{ V. anguillarum } \\
\hline NB10 & Wild-type, serotype O1, clinical isolate from the Gulf of Bothnia & Norqvist et al. (1989) \\
\hline $\mathrm{AC} 11$ & $\mathrm{NB} 10$ carrying an in-frame deletion of $v a n O$ & Croxatto et al. (2004) \\
\hline $\mathrm{AC} 11 \mathrm{c}$ & AC11 derivative in which the wild-type $v a n O$ gene was exchanged for the mutant allele & This study \\
\hline $\mathrm{AC} 12$ & NB10 carrying an in-frame deletion of $r p o S$ & This study \\
\hline OTR83 & NB10 carrying an in-frame deletion of $r p o N$ & O’Toole et al. (1997) \\
\hline BW11 & NB10 carrying an-frame deletion of $h f q$ & This study \\
\hline BW11c & BW11 derivative in which the wild-type $h f q$ gene was exchanged for the mutant allele & This study \\
\hline \multicolumn{3}{|c|}{ 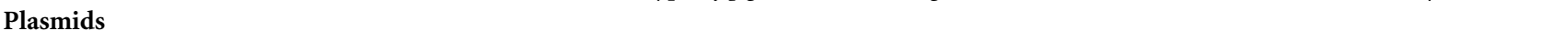 } \\
\hline pBluescript & Ap ${ }^{r}$; ColE1 origin & Stratagene \\
\hline pBS-rpoS-7 & $\begin{array}{l}\text { Ap }^{\mathrm{r}} \text {; pBluescript with a } \sim 9.5 \mathrm{~kb} \text { genomic fragment containing truD (partial), surE, } p c m \text {, } \\
n l p D, r p o S \text { and } m u t S \text { (partial) genes }\end{array}$ & This study \\
\hline pDM4-hfq-AD & $\mathrm{Cm}^{\mathrm{r}}$; pDM4 containing a mutant allele of $h f q$, bp 227-392 fused in-frame to bp 624-819 bp & This study \\
\hline pDM4-rpoS-wt & $\mathrm{Cm}^{\mathrm{r}}$; pDM4 containing the wild-type allele of $r p o S$ bp 2513-3874 & This study \\
\hline pDM4-vanO-wt & $\mathrm{Cm}^{\mathrm{r}}$; pDM4 containing the wild-type allele of $v a n O$ bp $635-1774$ & This study \\
\hline pDM4-hfq-wt & $\mathrm{Cm}^{\mathrm{r}}$; pDM4 containing the wild-type allele of $h f q$ bp $227-819$ & This study \\
\hline pNQ705-1 & $\mathrm{Cm}^{\mathrm{r}}$; suicide vector with an $\mathrm{R} 6 \mathrm{~K}$ origin (pir requiring) & McGee et al. (1996) \\
\hline pJBA113 & 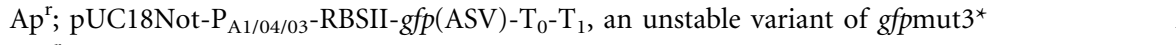 & Andersen et al. (1998) \\
\hline pDM41 & $\mathrm{Cm}^{\mathrm{r}}$; pNQ705-1 carrying the promoterless RBSII- $g f p(\mathrm{ASV})-\mathrm{T}_{0}$ gene from pJBA113 & This study \\
\hline pDM41-vanT3-TL & $\mathrm{Cm}^{\mathrm{r}} ;$ pDM41 carrying a $v a n T:: g f(\mathrm{ASV})$ translational gene fusion & This study \\
\hline pDM41-vanT-TC & $\mathrm{Cm}^{\mathrm{r}}$; pDM41 carrying a vanT::gfp(ASV) transcriptional gene fusion & This study \\
\hline pDM41-rpoS-TC & $\mathrm{Cm}^{\mathrm{r}}$; pDM41 carrying a $r p o S:: g f p(\mathrm{ASV})$ transcriptional gene fusion & This study \\
\hline pDM41-empA-TC & $\mathrm{Cm}^{\mathrm{r}}$; pDM41 carrying a empA::gfp(ASV) transcriptional gene fusion & This study \\
\hline
\end{tabular}

$25 \mu \mathrm{g} \mathrm{ml}^{-1}$ for chloramphenicol. For $V$. anguillarum, Trypticase soy medium from BBL containing $1 \%$ sodium chloride (TSB) was routinely used. For selection against $E$. coli after conjugation, the Vibrio selective medium TCBS agar (Difco) was used. Antibiotic concentration for $V$. anguillarum for chloramphenicol was $10 \mu \mathrm{g} \mathrm{ml}^{-1}$.

Cloning the rpos and $\boldsymbol{h f q}$ genes. An internal 477 bp DNA fragment from the $V$. anguillarum rpoS gene was amplified using PCR and degenerate primers complementary to the Vibrio cholerae rpoS gene VC0534 (Heidelberg et al., 2000) and sequenced to confirm identity to rpoS. The primers used were VCrpoS-For2 $\left(5^{\prime}-\right.$ GGACTAGTCCGTAACCAAAGTAGARGARTT-3') and VCrpoSRev2 (5'-GGGGTACCCCAATGGTGCGTGTYTGRTTCAT- ${ }^{\prime}$ ). The 477 bp PCR fragment was used as a probe to screen a $V$. anguillarum genomic library in the Lambda Zap II bacteriophage, and the pBluescript plasmids containing a chromosomal insert were excised from positive plaques as previously described (Milton et al., 1992). One plasmid that contained the entire rpoS gene, pBS-rpoS-7, was chosen for sequencing.

To clone the $h f q$ gene, two primers complementary to sequences flanking the $h f q$ gene in Vibrio parahaemolyticus (Makino et al., 2003) were used to amplify a $1519 \mathrm{bp}$ fragment from the $V$. anguillarum chromosome. Primers used were Hfq-1 (5'-GGACTAGTGCATCAACAACATGTAACAA- $3^{\prime}$ ), which contains a SpeI site at the $5^{\prime}$-end, and Hfq-2 (5'-CTCGAGCTCGGTTACCGACAGATGTGGGA-3'), which contains a SacI site at the $5^{\prime}$-end. This fragment was cloned into pBluescript using SacI and SpeI, creating pBS-hfq, and sequenced.
Mutant and complementation strains. To create a null mutation in $r p o S$ and $h f q$, an in-frame deletion was made by allelic exchange as described in detail previously (Milton et al., 1996). Plasmid pDM4rpoS-AD, which carries an altered allele of rpoS that encodes the first 14 amino acids fused to the last 61 amino acids of RpoS, was used to create strain $\mathrm{AC12}(\Delta r p o S)$. Plasmid pDM4-hfq-AD, carrying an altered allele of $h f q$ encoding the first five amino acids fused to the last five amino acids of $\mathrm{Hfq}$, was used to create strain BW11 $(\Delta h f q)$. The in-frame deletions were confirmed by sequencing a PCR-amplified DNA fragment of the deleted chromosomal locus and by Western blot analysis.

For complementation of the $\Delta r p o S, \Delta h f q$ and $\Delta v a n O$ mutations, reverse allelic exchange was done. For each, the wild-type gene and flanking DNA were amplified by PCR and cloned into pDM4, resulting in pDM4-rpoS-wt, pDM4-hfq-wt and pDM4-vanO-wt, which were used to exchange the mutant alleles for the wild-type gene, producing strains $\mathrm{AC} 12 \mathrm{c}, \mathrm{BW} 11 \mathrm{c}$ and $\mathrm{AC} 11 \mathrm{c}$, respectively. Complementation of the mutations was confirmed by Western blot analysis.

PCR conditions, DNA techniques, DNA sequencing and computer analyses. PCR was performed as previously described (McGee et al., 1996; Croxatto et al., 2002). Unless otherwise stated, all conditions for the various DNA techniques were as described by Sambrook et al. (1989). Reaction conditions for the DNA-modifying enzymes and DNA-restriction enzymes were performed as suggested by the manufacturers. Double-strand DNA sequencing was performed by 
automated sequencing on an ABI Prism 377 DNA sequencer and by primer walking in two directions from known regions of DNA sequence. DNA sequence editing was done using the Genetics Computer Group Sequence Analysis software (Devereux et al., 1984) of the Genetics Computer Group (University of Wisconsin). Database searches were done using the BLAST program from the National Center for Biotechnology Information. The sequence data have been submitted to the DDBJ/EMBL/GeneBank databases under accession number EU330190 for rpoS and EU330191 for $h f q$.

Western analysis and preparation of antisera. VanT, VanO, RpoS and Hfq were purified using the IMPACT T7 system from New England BioLabs. Genes encoding VanT, VanO, RpoS and Hfq were amplified by PCR and cloned into pTYB1 using NdeI and SapI, which fuses the coding region for the protein splicing element intein followed by a chitin-binding tag to the $3^{\prime}$-end of these genes. For optimal self-splicing, an extra glycine codon was added to the $3^{\prime}$-end of the vanT, rpoS and $h f q$ genes. Protein purification was performed as described by the manufacturer except for VanT cleavage from the chitin column, which was done for $48 \mathrm{~h}$. Using the purified proteins, polyclonal rabbit antisera were made by AgriSera $A B$, Sweden. Before use in Western analyses, the antisera were affinity purified using the MicroLink Protein Coupling kit (Pierce) via the manufacturer's instructions.

For each strain, Western blot analysis was performed on protein samples taken at various time points during growth. Proteins were separated as described by Laemmli (1970) using SDS-12.5\% PAGE and transferred to a nitrocellulose membrane (Schleicher and Schuell) using a SemiPhor semidry blotter (Hoefer TE 70 series). Enhanced chemiluminescence (ECL) Western blotting was performed according to the manufacturer's instructions (Amersham Life Sciences). As no suitable loading control was found that could be used to compare all time points during growth, two approaches were taken to determine equal sample loading. A second similarly loaded $12.5 \%$ PAGE was performed and stained with Coomassie blue. In addition, Western analysis was done using protein samples from two time points and two antisera to detect the protein of interest and the outer-membrane protein OmpU, which was used as a loading control. The intensities of the two protein bands were measured using QUANTITY ONE version 4.2.3 software (Bio-Rad) and the protein of interest was equalized to that of OmpU. The mutant to wild-type ratio was then determined.

Construction of transcriptional and translational gfp fusions. Transcriptional or translational reporter gene fusions with the gene encoding the unstable green fluorescent protein variant Gfp-ASV (Andersen et al., 1998) were made using the suicide vector pDM41. The C-terminal peptide tag ASV renders the stable Gfp protein susceptible to degradation by intracellular tail-specific proteases. The half-life of Gfp-ASV was determined to be $80 \mathrm{~min}$ in V. anguillarum (data not shown). To create pDM41, the $g f p$ gene, which lacks a promoter but has a ribosome-binding site (RBS), was removed from pJBA113 by NheI and XbaI digestion. The 850 bp fragment was purified from a $1 \%$ agarose gel using Ultrafree-DA spin columns (Millipore) and cloned into similar restriction sites of the suicide vector pNQ705-1. For transcriptional fusions, the $\mathrm{XbaI}$ site, which cleaves upstream of the RBS, was used and for translational fusions, the SphI site, which cleaves at the ATG start codon, was used. For transcriptional fusions of vanT, rpoS, empA, qrrl and $h f q$, DNA fragments containing promoters but lacking the possible RBS were amplified by PCR using the following primer sets containing either a SacI or an XbaI site at the 5'-ends: VanT-gfp-S (5'-CTCGAGCTCATTCGTTCCTGAACC-3') and VanT-gfp-X (5'-GCTCTAGAACTGTTGAATTGAGC-3'); RpoS-gfp-S (5'-CTCGAGCTCCCGTTGTCTATTCGG-3') and RpoS-gfp-X (5'-GCTCTAGAGAGCTAGCAAGACAT-3'); EmpA-gfp-1 (5'-CTCGAGCTCATATGCTCAACGAAC$\left.3^{\prime}\right)$ and EmpA-gfp-2 (5'-GCTCTAGAGTTATTATTAGCATC-3'); and Rnal-gfp-S (5'-CTCGAGCTCAGCAATATGAGGTCC-3') and Rnal-gfp-X (5'-GCTCTAGATATTGAATAGAGCAC-3'); Hfq-gfp-S $\left(5^{\prime}\right.$-CTCGAGCTCAAGCGTCAGATCACC- $\left.3^{\prime}\right)$ and Hfq-gfp-X (5'GCTCTAGAGTTGTAGTTATTTAG-3'). For a translational fusion of vanT, a DNA fragment containing the promoter, the RBS and codons for the first 14 amino acids was amplified by PCR using a primer set containing either a $S a c I$ or a $S p h I$ site at the $5^{\prime}$-end. The primer pair used was VanT-Sph3 (5'-GGACATGCATGCGTGATAAGCGAGTTC-3') and VanT-gfp-S (listed above). The DNA fragments were gel purified, digested with $S a c \mathrm{I} / \mathrm{Xba \textrm {I }}$ or $S a c \mathrm{I} / \mathrm{Sph} \mathrm{I}$ and ligated to a similarly digested pDM41, resulting in pDM41vanT3-TL, pDM41-vanT-TC, pDM41-rpoS-TC, pDM41-empA-TC, pDM41-hfq-TC and pDM41-qrr1-TC. All constructs were sequenced to ensure that the gene fusions were made properly. Each gene fusion was integrated into the promoter region of the respective gene on the chromosome. Since the promoters were duplicated on the chromosomes, these insertions did not disrupt the respective wild-type genes. Chromosomal integrations were checked by PCR analysis.

Green fluorescent protein (Gfp) assays. $V$. anguillarum cultures carrying the $g f p$ gene fusions were grown overnight at $24{ }^{\circ} \mathrm{C}$ in TSB with aeration. Overnight cultures were diluted to an $\mathrm{OD}_{600}$ of 0.001 in TSB and incubation was continued at $24{ }^{\circ} \mathrm{C}$ in TSB with aeration. At various time points during growth $(0,2,4,6,8,10,12,14,18,24 \mathrm{~h})$, samples were taken and the $\mathrm{OD}_{600}$ and numbers of c.f.u. were determined. To measure fluorescence at each time point, a cell number equivalent to an $\mathrm{OD}_{600}$ of 0.2 was removed from each sample and, when required, the samples were diluted to an $\mathrm{OD}_{600}$ of 0.2 in a final volume of $2 \mathrm{ml}$. This was done since the use of too many cells quenched the fluorescence output using a Bio-Rad VersaFluor fluorometer. To minimize any effects on gene expression due to the dilution of the cells, fluorescence was measured immediately following dilution with an excitation wavelength of $490 \mathrm{~nm}$ and an emission wavelength at $520 \mathrm{~nm}$, according to the manufacturer's instructions. The fluorescence units were then divided by an $\mathrm{OD}_{600}$ of 0.2 to obtain fluorescence relative to the cell number. The wild-type strain without a $g f p$ gene fusion treated similarly was used as a blank before taking the measurement. All measurements were done in triplicate and averaged.

UV irradiation survival. Overnight cultures of $V$. anguillarum grown in TSB at $24{ }^{\circ} \mathrm{C}$ with aeration were diluted in the same medium to an $\mathrm{OD}_{600}$ of 0.001 and incubated until an $\mathrm{OD}_{600}$ of $0.5\left(5 \times 10^{8}\right.$ cells $\mathrm{ml}^{-1}$ ) was reached. Bacteria were diluted in $4 \%$ artificial seawater

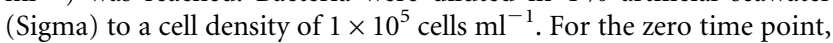
c.f.u. were measured from the culture used to inoculate each medium. Bacteria were exposed to shortwave UV light $(253.7 \mathrm{~nm})$ for various lengths of time and survival was measured by determining the c.f.u. in each sample. These assays were done at least three times.

RNA isolation and quantification. Overnight cultures of $V$. anguillarum grown at $24{ }^{\circ} \mathrm{C}$ with aeration were diluted in TSB to an $\mathrm{OD}_{600}$ of 0.001 and incubated until an $\mathrm{OD}_{600}$ of 1.0 was reached. Culture volumes equivalent to $1 \times 10^{8} \mathrm{cells} \mathrm{ml}^{-1}$ were mixed with 2 vols RNAprotect bacteria reagent (Qiagen) to stabilize the RNA transcripts and incubated for $5 \mathrm{~min}$ at room temperature. Total RNA was then isolated using the RNeasy minikit (Qiagen). RNA samples were treated with DNA-free DNase (Ambion) and RNA concentrations were then determined using the RiboGreen RNA reagent from Molecular Probes. Both were according to the manufacturers' instructions.

Real-time quantitative reverse transcriptase polymerase chain reaction (qRT-PCR). A one-step real-time qRT-PCR was used to measure the levels of vanT, qrrl and $h p d A$ mRNA in various $V$. anguillarum strains. RNA was purified from each strain as described above and $30 \mathrm{ng}$ was used in each RT-PCR reaction. An iCycler iQ 
(Bio-Rad) and the iScript One-Step RT-PCR kit with SYBR Green (Bio-Rad) were used according to the manufacturer's instructions. To ensure against contaminating chromosomal DNA in the purified RNA, one reaction using purified RNA as template for each sample was done using the iQ SYBR Green Supermix (Bio-Rad) according to the manufacturer's instructions. To ensure that equal amounts of RNA were used, one reaction contained primers for $16 S$ rRNA. RNA samples at each time point were prepared from three separate cultures. Primers used for each gene are as follows: vanT-right $\left(5^{\prime}\right.$ CTTTCGCATGCAAATCAAGA-3') and vanT-left (5'-CCACGCAGATATTGCTGAAA-3'); Qrr1-RT-Fw (5'-AAAGGTCTATTGGCTGTTATTTGTG- $\left.3^{\prime}\right)$ and Qrrl-RT-Rev (5'-ACCCTTAGGGGTCACCTAG-3'); HpdA-RT-Fw (5'-AGCATTCCTGCGATTTATGG-3') and HpdA-RT-Rev ( $5^{\prime}$-CGGTCATTCGTTGATTAGCA-3'); 16S-left (5'-CATGCCGCGTGTATGAAGAA- $3^{\prime}$ ) and 16S-right (5'-AACAATTATCGTCGTAGTAAACTGC- $3^{\prime}$ ). Calculations for mRNA levels were done according to the standard curve method (Larionov et al., 2005), which normalizes the mRNA levels to that of the 16S mRNA. Each qRT-PCR was done using samples from three independent experiments, the results were averaged, and $P$-values were determined.

vanT mRNA stability assay. Overnight cultures of $V$. anguillarum grown in TSB at $24{ }^{\circ} \mathrm{C}$ with aeration were diluted in the same medium to an $\mathrm{OD}_{600}$ of 0.001 and incubated to an $\mathrm{OD}_{600}$ of 0.2 and 1.0. To stop RNA transcription, rifampicin (Sigma-Aldrich) was added to the culture to a final concentration of $200 \mu \mathrm{g} \mathrm{ml}^{-1}$. For the zero time point, a culture sample $(100 \mu \mathrm{l})$ was taken before the addition of rifampicin. For mRNA half-life measurements, culture samples $(100 \mu \mathrm{l})$ were taken at 1, 2, 5 and $10 \mathrm{~min}$ after the addition of rifampicin. Total RNA was isolated from each sample and $30 \mathrm{ng}$ RNA was used in a real-time qRT-PCR as described above. For each strain, the zero time point was set to 1.0 and all following time points were normalized to the zero time point. To determine product specificity, standard curves and melting curves were analysed for multiple products. This assay was performed in triplicate.

\section{RESULTS}

\section{Expression of VanT during growth}

Previous studies suggested that the phosphorelay quorumsensing systems in $V$. anguillarum limit the expression of vanT rather than induce its expression (Croxatto et al., 2004). However, genes regulated by VanT are induced as the cell population enters stationary phase (Croxatto et al., 2002). Thus, we wondered whether VanT protein levels were induced during growth even though it was previously shown that the mRNA levels do not vary greatly. In the wild-type, Western blot analysis (Fig. 2) showed that VanT is detected at a low cell population $\left(\mathrm{OD}_{600} 0.007\right)$, indicating that the sRNAs do not completely destabilize vanT mRNA. An induction of expression was also seen as the cell population entered stationary phase $\left(\mathrm{OD}_{600} 0.4\right.$ 1.8), after which VanT levels decreased again. To determine if this induction is due to the quorum-sensing systems, VanT expression in a vanO mutant was analysed (Fig. 2). Compared to the wild-type, the vanO mutant showed increased levels of VanT at a low cell population $\left(\mathrm{OD}_{600}\right.$ $0.007-0.4$ ), indicating that the quorum-sensing systems repress VanT expression at low cell densities and the vanO mutation could be complemented with the wild-type gene. (a)

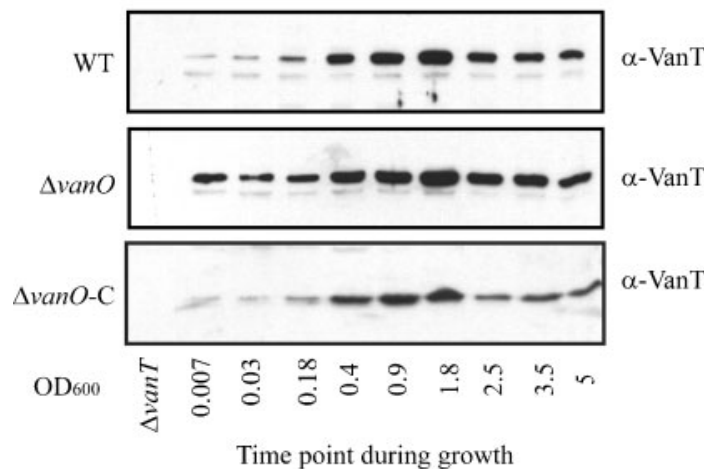

(b)

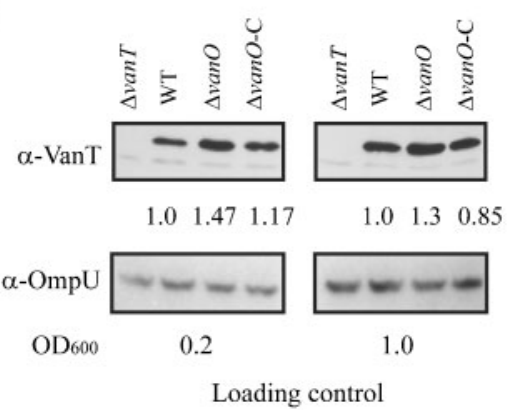

Fig. 2. Expression of $\operatorname{Van} T$ in the wild-type and the vanO mutant. (a) Culture samples of the wild-type (WT), vanO mutant $(\Delta v a n O)$ and a complemented vanO mutant $(\Delta v a n O-C)$ were taken at various cell densities. Proteins from equal cell numbers were separated by SDS-12.5\% PAGE and Western blot analyses were done using a VanT antiserum ( $\alpha$-VanT). As a negative control, the vanT mutant ( $\Delta$ vanT; sampled at $\left.\mathrm{OD}_{600} 1.0\right)$ was included. (b) OmpU was used as a loading/transfer control. Western blots were done as in (a) except that only two time points were analysed. After detection of VanT, the blot was stripped and an OmpU antiserum $(\alpha-\mathrm{OmpU})$ was applied. The intensities of the VanT and OmpU bands were measured and the intensity of VanT was equalized to that of OmpU. The mutant/wild-type ratio is given between the blots.

However, complete derepression of VanT expression at low cell density did not appear to occur in the vanO mutant and we wondered if an additional regulatory factor may induce the expression of VanT as cell numbers increase.

\section{RpoS regulates expression of VanT post- transcriptionally during entry into the stationary phase}

RpoS is a good candidate as an additional regulatory factor since it has been suggested to regulate the $V$. anguillarum empA gene, which encodes an extracellular metalloprotease that is also regulated by VanT (Croxatto et al., 2002; Denkin \& Nelson, 2004). Moreover, in E. coli, RpoS is the main regulator of gene expression during stationary phase (for a review see Hengge-Aronis, 2000). The rpoS genetic 
(a)
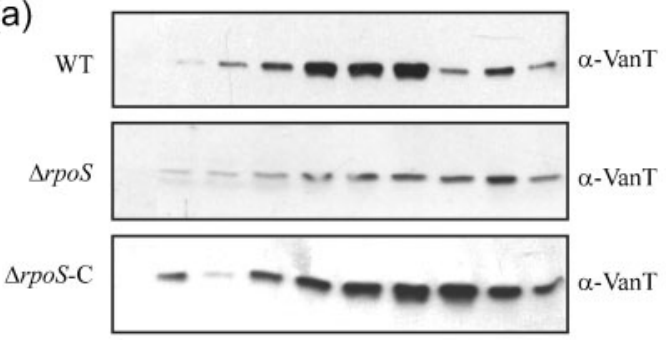

OD600 ڤิ

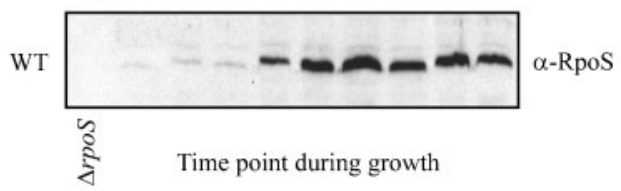

(b)

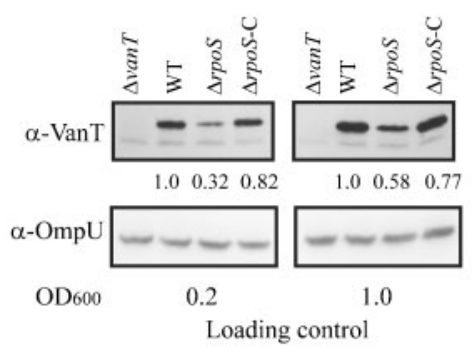

(c)

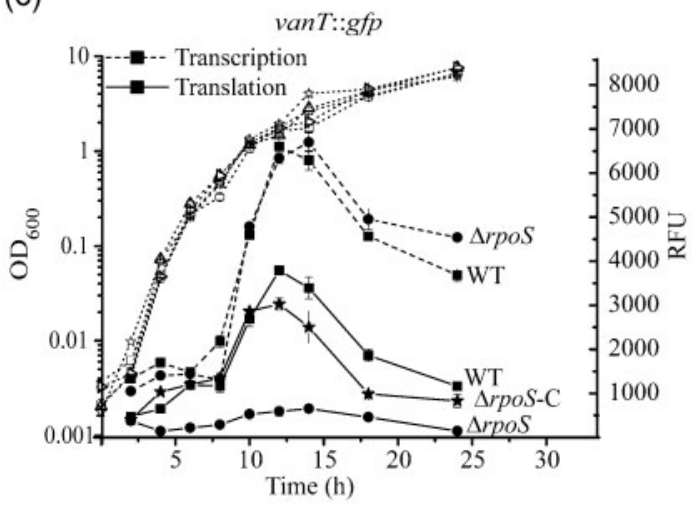

(d)

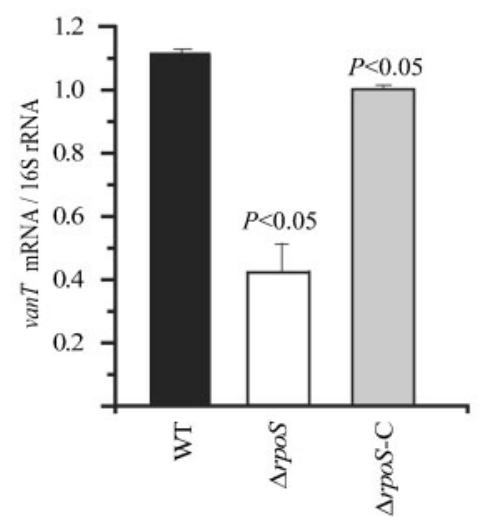

Fig. 3. RpoS regulates the expression of VanT post-transcriptionally. (a) Western blot analysis of VanT. Culture samples of the wildtype (WT), rpoS mutant $(\Delta r p o S)$ and a complemented rpoS mutant $(\Delta r p o S-C)$ were taken at various cell densities. Proteins from equal cell numbers were separated by SDS-12.5\% PAGE and Western blot analyses were done using a VanT antiserum for all three strains and an RpoS antiserum for the wild-type. As appropriate, the vanT mutant $(\Delta v a n T)$ and the rpoS mutant $(\Delta r p o S$; both sampled at $\mathrm{OD}_{600} 1.0$ ) were included as negative controls. (b) OmpU was used as a loading/transfer control. Western blots were done as in (a) except that only two time points were analysed. After detection of VanT the blot was stripped and an OmpU antiserum was applied. The intensities of the VanT and OmpU bands were measured and the VanT intensity was equalized to that of OmpU. The mutant/wild-type ratio is given between the blots. (c) A transcriptional (dashed lines) and a translational (solid lines) vanT::gfp gene fusion were expressed from the chromosome of the wild-type (WT, black squares), the rpoS mutant ( $\triangle$ rpoS, black circles) and the complemented rpoS mutant (ArpoS-C, black stars). Growth is indicated by dotted lines. Gfp expression was measured as fluorescence; relative fluorescence units (RFU) are fluorescence units of cells normalized to an $\mathrm{OD}_{600}$ of 0.2 . (d) Realtime qRT-PCR of vanT transcripts. RNA transcripts were isolated from the wild-type (WT), rpoS mutant ( $\Delta r p o S)$ and the complemented rpoS mutant ( $\Delta r p o S-C)$ grown to an $\mathrm{OD}_{600}$ of 1.0. Realtime qRT-PCR was done as described in Methods and vanT mRNA was normalized to the levels of $16 \mathrm{~S}$ RNA. $P$-values $<0.05$ are considered significant.

locus from $V$. anguillarum was cloned and sequenced. The RpoS amino acid sequence showed $73-86 \%$ identity with RpoS from other Vibrio species in the NCBI database. A mutant (AC12) carrying an in-frame deletion fusing the first 14 amino acids to the last 61 amino acids was made and the mutation was confirmed by Western analysis (data not shown).

To determine if VanT levels are altered in an rpoS mutant, Western analysis of VanT expression was done (Fig. 3a, b). The rpoS mutant did not show the peak of VanT expression during entry into stationary phase that occurred in the wild-type. To establish whether RpoS affects the transcription or translation of vanT, both types of gene fusions were made to a $g f p$ variant that encodes an unstable Gfp protein with a half-life of $80 \mathrm{~min}$ in $V$. anguillarum. Fluorescence was used as a measure of VanT expression throughout growth (Fig. 3c). For the transcriptional fusion, no difference was seen between the wild-type and the rpoS mutant. For the translational fusion, the induction of VanT expression seen during entry into stationary phase in the wild-type was lost in the rpoS mutant. In addition, using real-time qRT-PCR analysis, a decrease in vanT mRNA levels was seen in the rpoS mutant at an $\mathrm{OD}_{600}$ of 1.0 compared to the wild-type (Fig. 3d). For each assay, the loss of VanT expression seen in the rpoS mutant was restored to wild-type levels when the wild-type gene was exchanged for the mutant allele. Interestingly, the increase of VanT expression during late exponential growth 
(a)

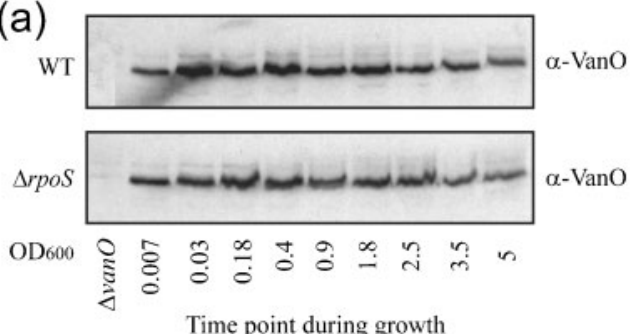

(b)

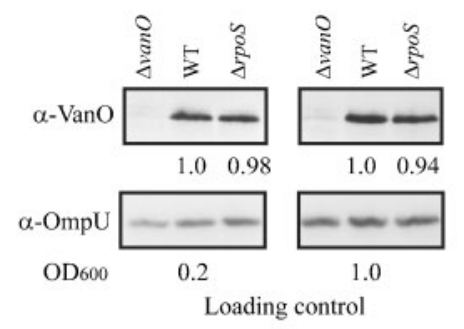

(c)

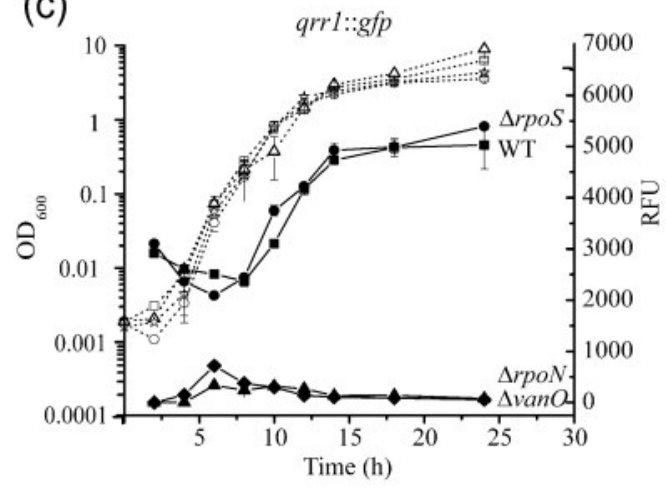

(d)

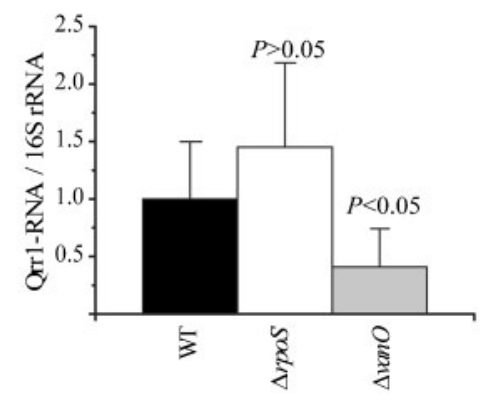

correlates with the onset of RpoS expression during growth (Fig. 3a). Together, these data suggest that RpoS indirectly regulates VanT expression via another gene product involved in the post-transcriptional regulation of vanT.

\section{RpoS regulation of vanT is not via the quorum- sensing regulatory cascade}

The data presented so far strongly suggest that RpoS regulates VanT expression independently of VanO and we wanted to confirm these observations. Based on other vibrio quorum-sensing systems (Lenz et al., 2004), VanO is
Fig. 4. RpoS regulation of vanT does not involve the quorumsensing regulatory cascade. (a) Western blot analysis of $\mathrm{VanO}$. Culture samples of the wild-type (WT) and the rpoS mutant ( $\Delta r p o S$ ) were taken at various cell densities. Proteins from equal cell numbers were separated by SDS-12.5\% PAGE and Western blot analysis was done using a $\mathrm{VanO}$ antiserum. The vanO mutant ( $\Delta v a n O$; sampled at $\mathrm{OD}_{600} 1.0$ ) was included as a negative control. (b) OmpU was used as a loading/transfer control. Western blots were done as in (a) except that only two time points were analysed. After detection of $\mathrm{VanO}$, the blot was stripped and an OmpU antiserum was applied. The intensities of the VanO and OmpU bands were measured and the $\mathrm{VanO}$ intensity was equalized to that of OmpU. The mutant/wild-type ratio is given between the blots. (c) A transcriptional qrr1::gfp gene fusion was expressed from the chromosome of the wild-type (WT, black squares), the rpoS mutant ( $\Delta r p o S$, black circles), the rpoN mutant ( $\triangle r p o N$, black diamonds) and the vanO mutant ( $\Delta v a n O$, black triangles). Growth is indicated by dotted lines. Gfp expression (solid lines) was measured as fluorescence; relative fluorescence units (RFU) are fluorescence units of cells normalized to an $\mathrm{OD}_{600}$ of 0.2. (d) Real-time qRT-PCR of grr1 transcripts. RNA transcripts were isolated from the wild-type $(\mathrm{WT}), r p o S$ mutant $(\Delta r p o S)$ and the vanO mutant $(\Delta v a n O)$ at $\mathrm{OD}_{600}$ 1.0. Real-time qRT-PCR was done as described in Methods. $P$ values $<0.05$ are considered significant.

believed to be required for the expression of the sRNAs that destabilize vanT mRNA. However, no difference in VanO levels was seen between the wild-type and the rpoS mutant (Fig. 4a, b). The Western blot analysis does not determine if the activity of VanO is affected. Thus, transcription analysis of an sRNA gene was done to measure the activity of VanO directly. To identify an sRNA gene for these studies, we used the fact that the sRNA Qrr1 (quorum-regulatory RNA) in other Vibrio species is encoded just upstream of luxO; DNA flanking the vanO gene was analysed for qrrl. Similarly in $V$. anguillarum, a qrr1 homologue with a consensus RpoN $\left(\sigma^{54}\right)$ binding site in the promoter region was found to be transcribed divergently from $v a n O$ (Fig. 1b). A qrr1::gfp transcriptional gene fusion was made and Gfp expression was assayed in the wild-type and in the vanO and rpoN mutants (Fig. 4c). In the wild-type, Gfp was expressed at low cell density and increased in expression during late exponential growth. In the rpoS mutant, Gfp expression from the qrrl promoter was unchanged compared to that of the wild-type; however, both $\mathrm{VanO}$ and $\mathrm{RpoN}$ were required for full expression. Real-time qRT-PCR analysis showed that qrr1 encodes a transcript in the wild-type and that the level of qrrl transcripts decreased in the vanO mutant compared to the wild-type but not in the rpoS mutant (Fig. 4d). Thus, the indirect post-transcriptional regulation of vanT by RpoS is not due to an effect on the expression or activation of VanO and thus expression of Qrr1.

\section{RpoS represses Hfq expression and stabilizes vanT MRNA}

From V. cholerae studies, Hfa, an RNA chaperone, was shown to work together with Qrr sRNAs to destabilize 

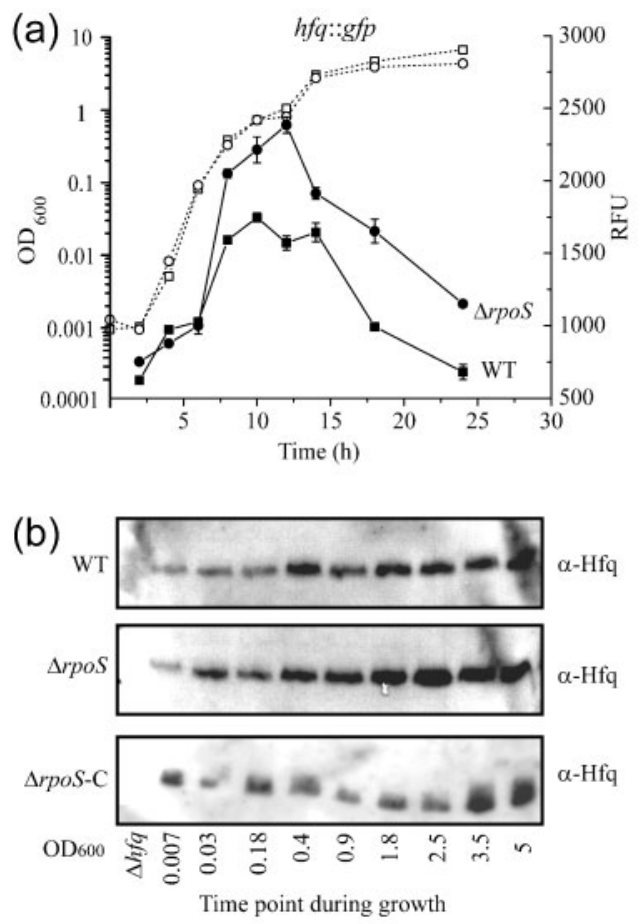

(c)

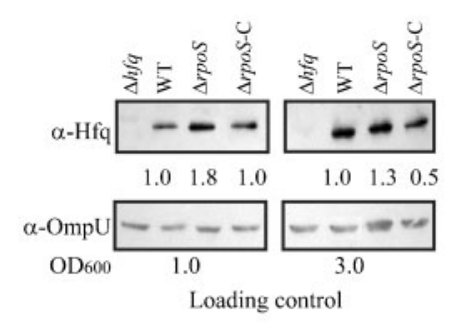

(d)

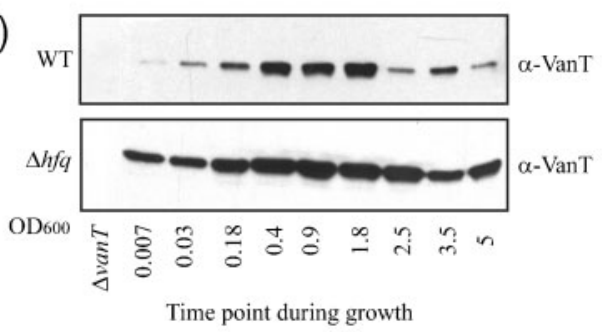

(e)

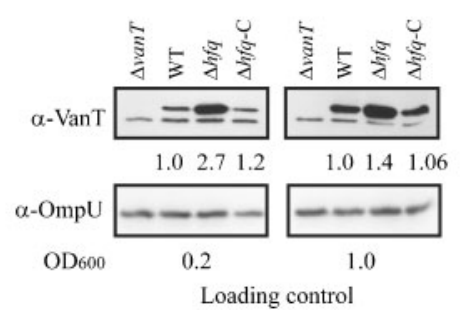

hapR mRNA (Lenz et al., 2004). Thus, we wondered if RpoS induces expression of VanT by affecting the expression of Hfq. The $h f q$ gene was cloned and the deduced amino acid sequence of Hfq showed 87-90\% identity with Hfq from other Vibrio species in the NCBI
Fig. 5. RpoS negatively affects expression of $\mathrm{Hfq}$ and $\mathrm{Hfq}$ represses vanT expression. (a) A transcriptional $h f q$ ::gfp gene fusion was expressed from the chromosome of the wild-type (WT; black squares) and the rpoS mutant ( $\Delta r p o S$, black circles). Growth is indicated by dotted lines. Gfp expression (solid lines) was measured as fluorescence; relative fluorescence units (RFU) are fluorescence units of cells normalized to an $\mathrm{OD}_{600}$ of 0.2 . (b) Western blot analysis of $\mathrm{Hfq}$ in an rpoS mutant. Culture samples of the wild-type (WT), the rpoS mutant $(\Delta r p o S)$ and the complemented rpoS mutant $(\Delta r p o S-C)$ were taken at various cell densities. Proteins from equal cell numbers were separated by SDS-12.5\% PAGE and Western blot analysis was done using an $\mathrm{Hfq}$ antiserum. The $h f q$ mutant ( $\Delta h f q$; sampled at $\left.\mathrm{OD}_{600} 1.0\right)$ was included as a negative control. (c) OmpU was used as a loading/ transfer control. Western blots were done as in (b) except that only two time points were analysed. After detection of $\mathrm{Hfq}$, the blot was stripped and an OmpU antiserum was applied. The intensities of the $\mathrm{Hfq}$ and $\mathrm{OmpU}$ bands were measured and the intensity of $\mathrm{Hfq}$ was equalized to that of $\mathrm{OmpU}$. The mutant/wild-type ratio is given between the blots. (d) Western blot analysis of VanT in an hfq mutant. Culture samples of the wild-type (WT) and the $h f q$ mutant $(\Delta h f q)$ were analysed for VanT expression as described in (b) except that the Western blot analysis was done using a VanT antiserum and the van $T$ mutant ( $\Delta$ van $T$; sampled at $\mathrm{OD}_{600} 1.0$ ) was included as a negative control. (e) Loading/transfer controls for (d) are as described for (c) except that a VanT antiserum was used and the complemented $h f q$ mutant strain $(\Delta h f q-\mathrm{C})$ was included.

database. An $h f q:: g f p$ transcriptional gene fusion was made and Gfp expression was assayed in the wild-type and $r p o S$ mutant (Fig. 5a). In the rpoS mutant, Gfp expression was similar to the wild-type during early growth. At the time point when RpoS is expressed in the wild-type (see Fig. 3), an increase in Gfp expression was seen in the rpoS mutant and Gfp expression remained higher than in the wild-type throughout growth. Western blot analysis showed a similar increase in Hfq levels in the rpoS mutant compared to the wild-type, and exchange of the mutant rpoS gene for the wild-type gene resulted in wild-type Hfq levels (Fig. 5b, c). To determine if an $h f q$ mutation affects VanT expression, an $h f q$ mutant carrying an in-frame deletion fusing the first five amino acids to the last five amino acids was made. In the $h f q$ mutant, VanT expression was derepressed throughout growth when compared to the wild-type (Fig. 5d, e).

Suggested roles for Hfq in gene regulation are to stabilize the sRNAs and to enhance RNA-RNA interactions (for a review see Gottesman, 2004). If an rpoS mutant has increased levels of $\mathrm{Hfq}$, then vanT mRNA would be predicted to be more unstable in this strain than in the wild-type. To test this hypothesis, the stability of vanT mRNA was determined in the wild-type and the rpoS, vanO and $h f q$ mutants at both a low and high cell density. Fig. 6 shows that at both cell densities vanT mRNA is more stable in the $h f q$ mutant than in the wild-type and the vanO mutant and, as expected, in the vanO mutant vanT mRNA is more stable than in the wild-type at low cell densities. However, vanT mRNA in the rpoS mutant, which has 
(a)

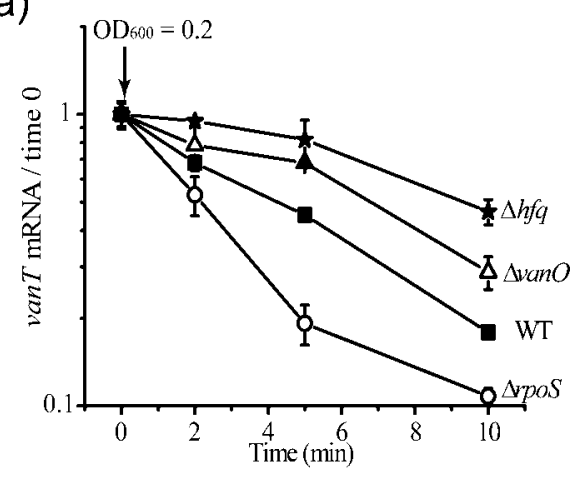

(b)

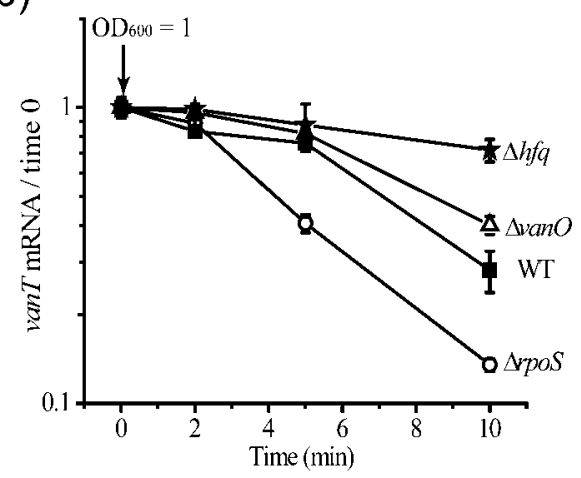

Fig. 6. Stability of van $T$ mRNA. The wild-type (black squares), the rpoS mutant $(\Delta r p o S$, white circles), the vanO mutant $(\Delta v a n O$, white triangles) and the $h f q$ mutant $(\Delta h f q$, black stars) were grown to an $\mathrm{OD}_{600}$ of 0.2 (a) and 1.0 (b). At these time points, transcription was stopped by the addition of rifampicin $(200 \mu \mathrm{g}$ $\mathrm{ml}^{-1}$ ) and culture samples were taken at 1, 2, 5 and $10 \mathrm{~min}$. The zero time point was taken before the addition of rifampicin. Total RNA was isolated from each sample and real-time qRT-PCR was done to determine the amount of vanT mRNA remaining at each time point. The mRNA level at the zero time point for each sample was set at 1.0 and the mRNA remaining at each time point after rifampicin addition was normalized to the respective zero time point.

increased levels of $\mathrm{Hfq}$, was much less stable than in the wild-type. These data show that Hfq and the Qrr sRNAs destabilize vanT mRNA and that RpoS increases the stability of vanT mRNA by negatively affecting the expression of $\mathrm{Hfq}$, resulting in an increase of VanT expression during late exponential growth.

\section{VanO and VanT do not affect expression of RpoS}

Quorum-sensing signalling in vibrios has been predicted to affect the levels of RpoS in the cell (McDougald \& Kjelleberg, 2006). To determine if the vibrio quorumsensing systems regulate expression of RpoS, Western blot analysis using an antiserum against RpoS was done in the wild-type and the vanO and vanT mutants. In addition, an $r p o S:: g f p$ transcriptional fusion was assayed in the same strains. No difference in RpoS expression was seen in either the vanO or the vanT mutant compared to the wild-type (Fig. 7), indicating that the quorum-sensing systems do not regulate RpoS expression.

\section{VanT and RpoS regulate similar cellular functions}

Studies in Vibrio vulnificus and V. cholerae showed that the vibrio quorum-sensing systems may play a role in bacterial physiology by regulating starvation adaptation and oxidative stress responses (McDougald et al., 2001, 2002; Joelsson et al., 2007). RpoS is a major transcriptional regulator essential for adaptation of bacteria to many stress responses during growth (for a review see Hengge-Aronis, 2000). In V. anguillarum, VanT may be part of the RpoS stress response. To test if VanT and RpoS are involved in stress response, the survival of bacterial cells from the wildtype and the rpoS and vanT mutants that were entering stationary phase was measured after exposure to UV irradiation for $45 \mathrm{~s}$. Resistance to UV irradiation, which damages DNA or induces the production of reactive oxygen species, is a likely stress response for many marine vibrios that are exposed to sunlight. Cells from both mutants showed a 100-fold decrease in survival compared to the wild-type and this phenotype could be complemented in the rpos mutant with the wild-type rpos gene (Fig. 8a).

Previously, we showed that VanT regulates gene expression during entry into stationary phase. Two genes identified were $h p d A$, which encodes a 4-hydroxyphenylpyruvate dioxygenase that is involved in pigment production, and empA, which encodes an extracellular metalloprotease (Croxatto et al., 2002). We asked whether RpoS regulates these genes as well. Using real-time qRT-PCR, a decrease in the $h p d A$ mRNA was seen in the vanT and rpoS mutants as compared to the wild-type (Fig. 8b). Expression of EmpA, which was measured using an empA::gfp transcriptional fusion, required both RpoS and VanT as no or very little Gfp expression was detected in either of the two mutants (Fig. 8c). Expression of both genes returned to approximately wild-type levels in the rpos mutant when the mutation was complemented with the wild-type rpoS gene. These data show that RpoS and VanT regulate similar physiological responses.

\section{DISCUSSION}

For Vibrio species studied so far, vibrio-specific phosphorelay quorum-sensing systems are a main regulatory pathway for modulating expression of $V$. harveyi LuxRtype transcriptional activators (reviewed by Milton, 2006; McDougald \& Kjelleberg, 2006). These quorum-sensing systems tightly repress expression of LuxR-type activators at low cell density. As the bacterial population grows, signal molecules accumulate and repress the phosphorylation relay, resulting in derepressed expression of the LuxR homologues. Previously, we showed that at low cell density, 
(a)

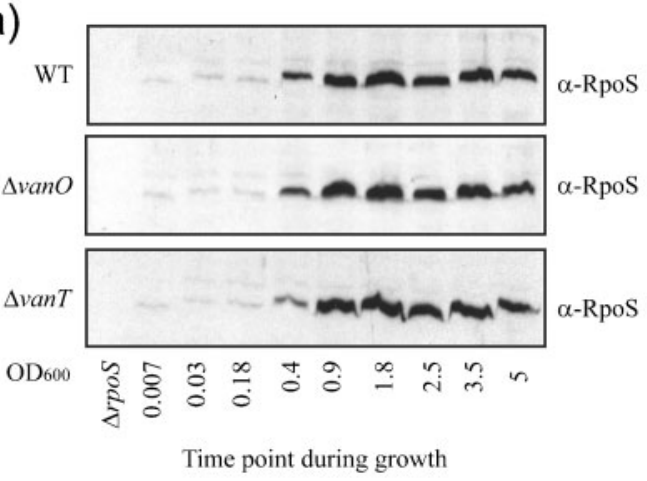

(b)

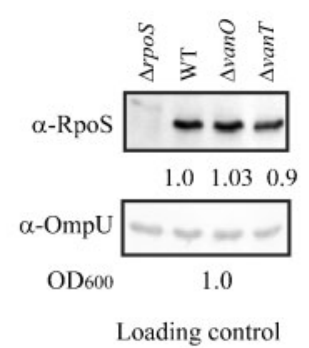

(c)

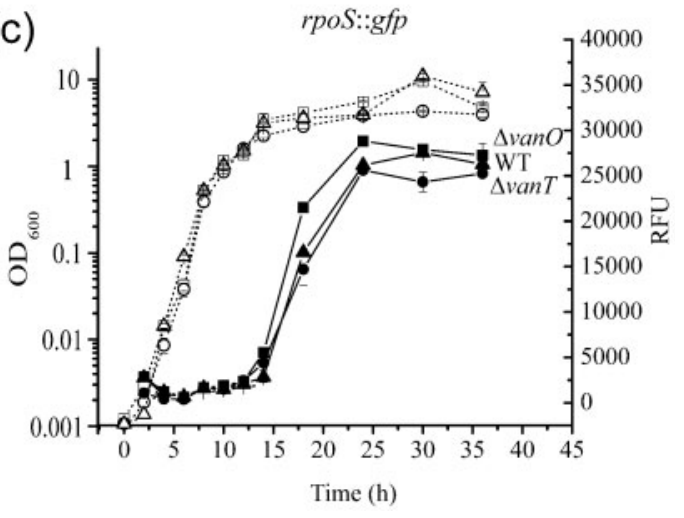

Fig. 7. VanO and VanT do not affect expression of rpoS. (a) Western blot analyses of RpoS were done using the wild-type $(\mathrm{WT})$, the vanO mutant $(\Delta \operatorname{vanO})$ and the vanT mutant $(\Delta \operatorname{van} T)$. Culture samples were taken at various cell densities. Proteins from equal cell numbers were separated by SDS-12.5\% PAGE and Western blot analyses were done using an RpoS antiserum. The rpoS mutant ( $\triangle r p o S$; sampled at $\left.\mathrm{OD}_{600} 1.0\right)$ was included as a negative control. (b) OmpU was used as a loading/transfer control for the Western blots. Western blots were done as in (a) except that only one time point was analysed. After detection of RpoS, the blot was stripped and an OmpU antiserum was applied. The intensities of the RpoS and OmpU bands were measured and the intensity of RpoS was equalized to that of OmpU. The mutant/wildtype ratio is given between the blots. (c) A transcriptional rpoS::gfp gene fusion was expressed from the chromosome of the wild-type (WT, black squares), the van $T$ mutant ( $\Delta$ van $T$, black circles) and the vanO mutant ( $\Delta$ vanO, black triangles). Growth is indicated by dotted lines. Gfp expression (solid lines) was measured as fluorescence; relative fluorescence units (RFU) are fluorescence units of cells normalized to an $\mathrm{OD}_{600}$ of 0.2 . similar quorum-sensing systems in $V$. anguillarum do not completely repress the mRNA levels of vanT, which encodes a LuxR-type transcriptional activator (Croxatto et al., 2004). This observation led to the suggestion that these regulatory systems limit rather than tightly repress the expression of VanT.

In the present study, we further characterized the expression of VanT in $V$. anguillarum. As predicted from other vibrio systems, $\mathrm{VanO}$ was shown to be required for expression of the sRNA Qrr1 and thus likely activates expression of other qrr genes seen in other Vibrio species but not yet identified in $V$. anguillarum. Previously, vanT mRNA was shown to be abundant in the wild-type; however, a vanO mutant showed a twofold increase in vanT mRNA, suggesting that repression of VanT expression does occur via the quorum-sensing regulation (Croxatto et al., 2004). Here, vanT mRNA was shown to be more stable in a vanO mutant and significantly more stable in an $h f q$ mutant than in the wild-type. These data suggest that vanT mRNA destabilization occurs via the sRNAs induced by VanO, that sRNAs work with the RNA chaperone $\mathrm{Hfq}$ to destabilize vanT mRNA, and that additional unidentified Qrr sRNAs are also likely involved as is the case for $V$. harveyi and $V$. cholerae (Lenz et al., 2004; Tu \& Bassler, 2007). VanO has less of an effect on destabilizing vanT mRNA than Hfq since it may not be absolutely required for expression of the qrr sRNA genes. A low level of qrr1 expression was seen in the absence of VanO (Fig. 4). Thus, V. anguillarum utilizes the sRNAs induced by VanO to destabilize vanT mRNA; however, repression of VanT expression is not as tight as in other vibrios.

In $V$. anguillarum, the Qrr sRNAs may be less effective at destabilizing vanT mRNA than Qrr sRNAs in other Vibrio species. One possible reason is that the affinity of the Qrr sRNAs for vanT mRNA is less than that in other vibrios. Moreover, other target mRNAs may exist for the Qrr1 sRNA that are not yet identified and that may be better targets for Qrr1 compared to vanT mRNA. Recently, $V$. cholerae was shown to have additional target mRNAs other than hapR mRNA that are regulated by the Qrr sRNAs (Hammer \& Bassler, 2007). A second explanation may be that the Qrr sRNAs may compete with other sRNAs or RNA-binding proteins for binding to vanT mRNA. One speculation is that since VanU was shown to activate instead of repress VanT expression, VanU may play a pivotal role in VanT expression by creating a balance between repression and activation (Croxatto et al., 2004). To do this, VanU may activate a second response regulator not yet identified that induces the expression of additional sRNAs or an RNA-binding protein that can interfere with Qrr sRNA binding.

This putative balance between repression and activation of VanT expression may easily be influenced by other elements that affect the sRNAs or the response regulator, or possibly by other regulatory elements that act indepen- 
(a)

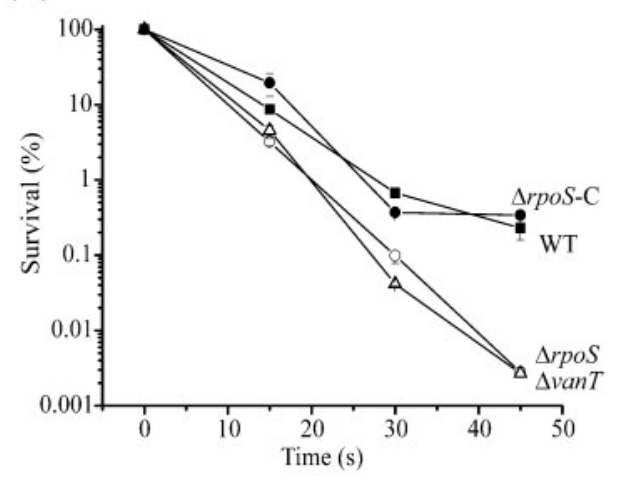

(b)

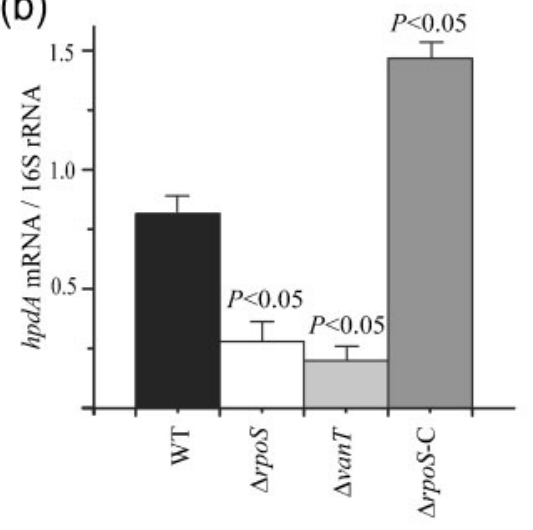

(c)

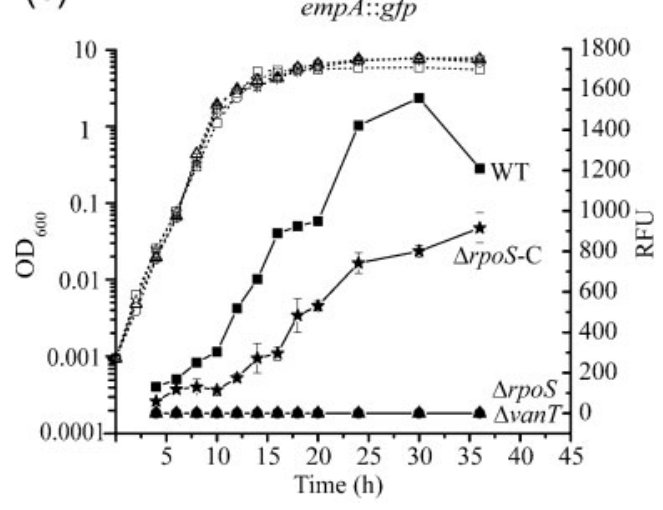

dently of the quorum-sensing phosphorelay mechanism. As shown in this study, RpoS induced post-transcriptionally the expression of VanT at high cell density by negatively affecting $\mathrm{Hfq}$ expression. Consequently, vanT mRNA was less stable in an rpoS mutant than in the wildtype (Fig. 6). It is also likely that RpoS may affect vanT expression by other mechanisms not yet characterized. Interestingly, VanO and VanT did not regulate RpoS and RpoS did not regulate $\mathrm{VanO}$, suggesting that RpoS works independently of the quorum-sensing system to regulate VanT. A model for regulation of vanT expression is given in Fig. 9. The role of RpoS in quorum sensing in vibrios differs. In $V$. harveyi, RpoS is not involved in regulation of luminescence (Lin et al., 2002). In V. vulnificus, RpoS does
Fig. 8. VanT and RpoS regulate similar cellular functions. (a) UV irradiation survival. Cultures of the wild-type (WT, black squares), the rpoS mutant ( $\Delta r p o S$, open circles), the complemented rpoS mutant $(\Delta r p o S-C$, black circles) and the van $T$ mutant ( $\Delta$ van $T$, open triangles) that were entering stationary phase were irradiated with UV light for various times. Aliquots $\left(10^{5}\right.$ cells $\left.\mathrm{ml}^{-1}\right)$ of each culture were removed at various time points during exposure and c.f.u. counts were determined. The results are given as percentage survival of the initial cell density at time zero. (b) Real-time qRT$\mathrm{PCR}$ of $h p d A$ transcripts. RNA transcripts were isolated from the wild-type (WT), rpoS mutant $(\Delta r p o S)$, the complemented rpoS mutant $(\Delta r p o S-C)$ and the vanT mutant $(\Delta v a n T)$ at an $\mathrm{OD}_{600}$ of 1.0. Real-time qRT-PCR was done as described in Methods. $P$ values $<0.05$ are considered significant. (c) A transcriptional empA::gfp gene fusion was expressed from the chromosome of the wild-type (WT, black squares), the rpoS mutant ( $\Delta r p o S$, black triangles), the complemented rpoS mutant ( $\Delta r p o S-\mathrm{C}$, black stars) and as a control, the van $T$ mutant ( $\Delta$ van $T$, black circles). Growth is indicated by dotted lines. Gfp expression (solid lines) was measured as fluorescence; relative fluorescence units (RFU) are fluorescence units of cells normalized to an $\mathrm{OD}_{600}$ of 0.2 .

not affect the expression of SmcR, a LuxR homologue, and vice versa (Jeong et al., 2003). In V. cholerae, RpoS represses LuxO expression during exponential growth, which enhances derepression of HapR expression via the quorum-sensing regulatory systems (Yildiz et al., 2004; Nielsen et al., 2006). In addition, HapR enhances expression of RpoS, suggesting a possible autoregulation loop in this bacterium (Joelsson et al., 2007).

For many bacteria, quorum-sensing regulation is integrated within other global regulatory networks. Signal molecule accumulation is not always sufficient for induction of quorum-sensing-regulated genes. Additional regulatory factors are required that allow the bacteria a second check before inducing a large number of genes that will be energetically costly (Schuster \& Greenberg, 2006). A well-studied example of this type of integration is the relationship between RpoS, the global regulator for bacterial adaptation to stress responses (for a review see Hengge-Aronis, 2000), and quorum-sensing regulation in Pseudomonas aeruginosa (Schuster et al., 2004). Transcriptome analyses showed that RpoS regulates, directly or indirectly, more than $40 \%$ of all quorum-sensing-regulated genes. Moreover, the quorum-sensing transcriptional regulators LasR and RhlR weakly induce expression of RpoS and vice versa. Schuster et al. (2004) proposed a model for the interaction between these two global regulatory networks by grouping the regulated genes into classes. Some genes are directly regulated by RpoS but indirectly regulated by quorum sensing, which weakly regulates rpoS. Other genes are directly regulated by quorum sensing and indirectly regulated by RpoS, which weakly regulates $l a s R$ and $r h l R$. However, some genes are directly regulated by both RpoS and quorum sensing. Although not as well studied as in $P$. aeruginosa, the integration of RpoS regulation with quorum-sensing 


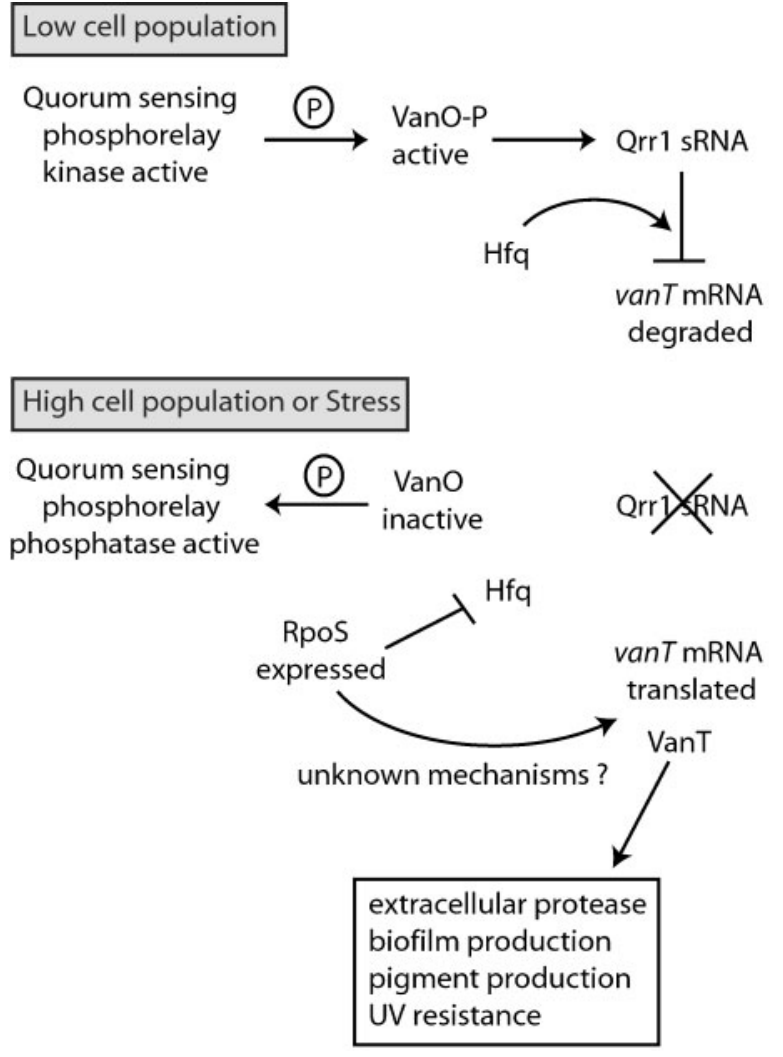

Fig. 9. Model for regulation of VanT expression. At a low cell population, quorum-sensing signal molecules are present in very low concentrations if at all. $\mathrm{VanO}$, which induces expression of at least one sRNA Orr1, is phosphorylated and activated by the quorum-sensing phosphorelay. Small RNAs together with the RNA chaperone $\mathrm{Hfq}$ aid degradation of vanT mRNA, greatly reducing the expression of VanT and all genes activated by VanT. At a high cell population, two independent regulatory systems activate vanT expression. First, quorum-sensing signal molecules enable a phosphatase activity to predominate in the phosphorelay, which leads to dephosphorylation and inactivation of $\mathrm{VanO}$. Consequently, sRNAs are not transcribed and VanT expression is induced. Second, RpoS induces VanT expression posttranscriptionally by inhibiting expression of $\mathrm{Hfq}$ and possibly via other mechanisms not yet identified. Since RpoS is a main regulator of gene expression during stress response, we suggest that stress response will also induce VanT expression.

regulation is seen with other bacteria as well, for instance Burkholderia cepacia (Aguilar et al., 2003), Ralstonia solanacearum (Flavier et al., 1998) and Erwinia carotovora (Mukherjee et al., 2000).

An interesting observation in this study is that Qrr1 expression in $V$. anguillarum was activated throughout growth and the expression increased during late exponential growth instead of decreasing as was seen for the qrr genes in V. harveyi ( $\mathrm{Tu} \&$ Bassler, 2007). Continual expression of Qrr1 during growth is likely due to VanO since Qrr1 expression required VanO throughout growth.
If this is true, it opens up the possibility that VanO is phosphorylated and active at high cell density as well as at low cell density, possibly by a mechanism other than the quorum-sensing systems. Alternatively a more complex regulatory mechanism is likely required for the increase in Qrr1 expression during late exponential growth that involves $\mathrm{VanO}$ and possibly another unidentified transcriptional regulator. Further studies are required to determine why Qrr1 expression increases as VanT expression increases and as the bacterial cell population increases.

Taken together these data suggest that VanT expression appears to respond both to the cell population via quorum-sensing regulation and to stress responses via RpoS regulation. In E. coli and P. aeruginosa (HenggeAronis, 2000; Schuster et al., 2004) the RpoS induction is suggested to occur during stress responses, such as those to heat, osmotic and oxidative stress, irrespective of the growth phase. VanT and RpoS regulate similar cellular functions, suggesting that VanT is part of the RpoS regulon in $V$. anguillarum. VanT function can thus be linked to the bacterial stress response and general physiology. Vibriospecific quorum-sensing systems have previously been suggested to play a role in physiology by regulating starvation adaptation and stress responses (McDougald et al., 2001, 2002). V. anguillarum is widely distributed in the aquatic environment from fresh to deep-sea waters (Urakawa \& Rivera, 2006) and forms biofilms on abiotic surfaces in seawater and on fish skin tissue (Tait et al., 2005; Croxatto et al., 2007). Quorum sensing is likely required to coordinate and to perform numerous physiological activities needed for the bacteria to survive as a population in the highly variable aquatic environment.

\section{ACKNOWLEDGEMENTS}

This work was performed within the Umeå Centre for Microbial Research (UCMR). We would like to thank Andrea Hardman for technical assistance during the cloning of rpoS and Udo Bläsi for antiserum to Hfq. This work was supported by grants from the Swedish Council for Environment, Agricultural Sciences and Spatial Planning, from the Carl Tryggers Foundation, Sweden, from the Swedish Research Council and from The Wenner-Gren Foundation, which are gratefully acknowledged.

\section{REFERENCES}

Actis, L. A., Tolmasky, M. E. \& Crosa, J. H. (1999). Vibriosis. In Fish Diseases and Disorders, vol. 3, Viral, Bacterial and Fungal Infections, pp. 523-557. Edited by P. T. K. Woo \& E. W. Bruno. Wallingford: CABI.

Aguilar, C., Bertani, I. \& Venturi, V. (2003). Quorum-sensing system and stationary-phase sigma factor $(r p o S)$ of the onion pathogen Burkholderia cepacia genomovar I type strain, ATCC-25416. Appl Environ Microbiol 69, 1739-1747.

Andersen, J. B., Sternberg, C., Poulsen, L. K., Bjørn, S. P., Givskov, M. \& Molin, S. (1998). New unstable variants of green fluorescent protein for studies of transient gene expression in bacteria. Appl Environ Microbiol 64, 2240-2246. 
Austin, B. \& Austin, D. A. (1999). Pathogenicity. In Bacterial Fish Pathogens: Disease of Farmed and Wild Fish, pp. 272-275. Chichester: Springer-Praxis.

Barrios, H., Valderrama, B. \& Morett, E. (1999). Compilation and analysis of $\sigma^{54}$-dependent promoter sequences. Nucleic Acids Res 27, 4305-4313.

Buch, C., Sigh, J., Nielsen, J., Larsen, J. L. \& Gram, L. (2003). Production of acylated homoserine lactones by different serotypes of Vibrio anguillarum both in culture and during infection of rainbow trout. Syst Appl Microbiol 26, 338-349.

Croxatto, A., Chalker, V. J., Lauritz, J., Jass, J., Hardman, A., Williams, P., Cámara, M. \& Milton, D. L. (2002). VanT, a homologue of Vibrio harveyi LuxR, regulates serine, metalloprotease, pigment, and biofilm production in Vibrio anguillarum. J Bacteriol 184, 1617-1629.

Croxatto, A., Pride, J., Hardman, A., Williams, P., Cámara, M. \& Milton, D. L. (2004). A distinctive dual-channel quorum-sensing system operates in Vibrio anguillarum. Mol Microbiol 52, 1677-1689.

Croxatto, A., Lauritz, J., Chen, C. \& Milton, D. L. (2007). Vibrio anguillarum colonization of rainbow trout integument requires a DNA locus involved in exopolysaccharide transport and biosynthesis. Environ Microbiol 9, 370-382.

Denkin, S. M. \& Nelson, D. R. (2004). Regulation of Vibrio anguillarum empA metalloprotease expression and its role in virulence. Appl Environ Microbiol 70, 4193-4202.

Devereux, J., Haeberli, P. \& Smithies, O. (1984). A comprehensive set of sequence analysis programs for the VAX. Nucleic Acids Res 12, 387-395

Flavier, A. B., Schell, M. A. \& Denny, T. P. (1998). An RpoS $\left(\sigma^{\mathrm{S}}\right)$ homologue regulates acylhomoserine lactone-dependent autoinduction in Ralstonia solanacearum. Mol Microbiol 28, 475-486.

Gottesman, S. (2004). The small RNA regulators of Escherichia coli: roles and mechanisms. Annu Rev Microbiol 58, 303-328.

Hammer, B. K. \& Bassler, B. L. (2007). Regulatory small RNAs circumvent the conventional quorum sensing pathway in pandemic Vibrio cholerae. Proc Natl Acad Sci U S A 104, 11145-11149.

Heidelberg, J. F., Eisen, J. A., Nelson, W. C., Clayton, R. A., Gwinn, M. L., Dodson, R. J., Haft, D. H., Hickey, E. K., Peterson, J. D. \& other authors (2000). DNA sequence of both chromosomes of the cholera pathogen Vibrio cholerae. Nature 406, 477-484.

Hengge-Aronis, R. (2000). The general stress response in Escherichia coli. In Bacterial Stress Responses, pp. 161-178. Edited by G. Storze \& R. Hengge-Aronis. Washington, DC: American Society of Microbiology.

Henke, J. M. \& Bassler, B. L. (2004). Three parallel quorum-sensing systems regulate gene expression in Vibrio harveyi. J Bacteriol 186, 6902-6914.

Higgins, D. A., Pomianek, M. E., Kraml, C. M., Taylor, R. K., Semmelhack, M. F. \& Bassler, B. L. (2007). The major Vibrio cholerae autoinducer and its role in virulence factor production. Nature 450, 883-886.

Jeong, H. S., Lee, M. H., Lee, K.-H., Park, S.-J. \& Choi, S. H. (2003). SmcR and cyclic AMP receptor protein coactivate Vibrio vulnificus $v v p E$ encoding elastase through the RpoS-dependent promoter in a synergistic manner. J Biol Chem 278, 45072-45081.

Joelsson, A., Kan, B. \& Zhu, J. (2007). Quorum sensing enhances stress response in Vibrio cholerae. Appl Environ Microbiol 73, 3742-3746.

Laemmli, U. K. (1970). Cleavage of structural proteins during the assembly of the head of bacteriophage T4. Nature 227, 680-685.

Larionov, A., Krause, A. \& Miller, W. (2005). A standard curve based method for relative real time PCR data processing. $B M C$ Bioinformatics 6, 62.
Lenz, D. H., Mok, K. C., Lilley, B. N., Kulkarni, R. V., Wingreen, N. S. \& Bassler, B. L. (2004). The small RNA chaperone Hfq and multiple small RNAs control quorum sensing in Vibrio harveyi and Vibrio cholerae. Cell 118, 69-82.

Lin, Y. H., Miyamoto, C. \& Meighen, E. A. (2002). Cloning, sequencing, and functional studies of the rpoS gene from Vibrio harveyi. Biochem Biophys Res Commun 293, 456-462.

Makino, K., Oshima, K., Kurokawa, K., Yokoyama, K., Uda, T., Tagomori, K., lijima, Y., Najima, M., Nakano, M. \& other authors (2003). Genome sequence of Vibrio parahaemolyticus: a pathogenic mechanism distinct from that of $V$. cholerae. Lancet 361, 743-749.

McDougald, D. \& Kjelleberg, S. (2006). Adaptive responses of vibrios. In The Biology of Vibrios, pp. 133-155. Edited by F. L. Thompson, B. Austin \& J. Swings. Washington, DC: American Society for Microbiology.

McDougald, D., Rice, S. A. \& Kjelleberg, S. (2001). SmcR-dependent regulation of adaptive phenotypes in Vibrio vulnificus. J Bacteriol 183, 758-762.

McDougald, D., Gong, L., Srinivasan, S., Hild, E., Thompson, L., Takayama, K., Rice, S. A. \& Kjelleberg, S. (2002). Defences against oxidative stress during starvation in bacteria. Antonie Van Leeuwenhoek 81, 3-13.

McGee, K., Hörstedt, P. \& Milton, D. L. (1996). Identification and characterization of additional flagellin genes from Vibrio anguillarum. $J$ Bacteriol 178, 5188-5198.

Milton, D. L. (2006). Quorum sensing in vibrios: complexity for diversification. Int J Med Microbiol 296, 61-71.

Milton, D. L., Norqvist, A. \& Wolf-Watz, H. (1992). Cloning of a metalloprotease gene involved in the virulence mechanism of Vibrio anguillarum. J Bacteriol 174, 7235-7244.

Milton, D. L., O'Toole, R., Hörstedt, P. \& Wolf-Watz, H. (1996). Flagellin A is essential for the virulence of Vibrio anguillarum. $J$ Bacteriol 178, 1310-1319.

Mukherjee, A., Cui, Y., Ma, W., Liu, Y. \& Chatterjee, A. K. (2000). hexA of Erwinia carotovora ssp. carotovora strain Ecc71 negatively regulates production of RpoS and rsmB RNA, a global regulator of extracellular proteins, plant virulence and the quorum-sensing signal, $\mathrm{N}$-(3oxohexanoyl)-L-homoserine lactone. Environ Microbiol 2, 203-215.

Neiditch, M. B., Federle, M. J., Miller, S. T., Bassler, B. L. \& Hughson, F. M. (2005). Regulation of LuxPQ receptor activity by the quorumsensing signal autoinducer-2. Mol Cell 18, 507-518.

Nielsen, A. T., Dolganov, N. A., Otto, G., Miller, M. C., Wu, C. Y. \& Schoolnik, G. K. (2006). RpoS controls the Vibrio cholerae mucosal escape response. PLoS Pathog 2, e109.

Norqvist, A., Hagström, Å. \& Wolf-Watz, H. (1989). Protection of rainbow trout against vibriosis and furunculosis by the use of attenuated strains of Vibrio anguillarum. Appl Environ Microbiol 55, 1400-1405.

O’Toole, R., Milton, D. L., Hörstedt, P. \& Wolf-Watz, H. (1997). RpoN of the fish pathogen Vibrio (Listonella) anguillarum is essential for flagellum production and virulence by the water-borne but not intraperitoneal route of inoculation. Microbiology 143, 3849-3859.

Sambrook, J., Fritsch, E. F. \& Maniatis, T. (1989). Molecular Cloning: a Laboratory Manual, 2nd edn. Cold Spring Harbor, NY: Cold Spring Harbor Laboratory.

Schuster, M. \& Greenberg, E. P. (2006). A network of networks: quorum-sensing gene regulation in Pseudomonas aeruginosa. Int $\mathrm{J}$ Med Microbiol 296, 73-81.

Schuster, M., Hawkins, A. C., Harwood, C. S. \& Greenberg, E. P. (2004). The Pseudomonas aeruginosa RpoS regulon and its relationship to quorum sensing. Mol Microbiol 51, 973-985. 
Tait, K., Joint, I., Daykin, M., Milton, D. L., Williams, P. \& Cámara, M. (2005). Disruption of quorum-sensing in seawater abolishes attraction of zoospores of the green alga Ulva to bacterial biofilms. Environ Microbiol 7, 229-240.

Timmen, M., Bassler, B. L. \& Jung, K. (2006). AI-1 influences the kinase activity but not the phosphatase activity of LuxN of Vibrio harveyi. J Biol Chem 281, 24398-24404.

Tu, K. C. \& Bassler, B. L. (2007). Multiple small RNAs act additively to integrate sensory information and control quorum sensing in Vibrio harveyi. Genes Dev 21, 221-233.
Urakawa, H. \& Rivera, I. N. G. (2006). Aquatic environment. In The Biology of Vibrios, pp. 175-189. Edited by F. L. Thompson, B. Austin \& J. Swings. Washington, DC: American Society for Microbiology.

Waters, C. M. \& Bassler, B. L. (2005). Quorum sensing: cell-to-cell communication in bacteria. Annu Rev Cell Dev Biol 21, 319-346.

Yildiz, F. H., Liu, X. S., Heydorn, A. \& Schoolnik, G. K. (2004). Molecular analysis of rugosity in a Vibrio cholerae O1 El Tor phase variant. Mol Microbiol 53, 497-515.

Edited by: P. Cornelis 